\title{
Identificación y localización de fallas en sistemas de distribución, utilizando medidores de calidad del servicio de energía eléctrica ${ }^{1}$
}

\author{
Identification and troubleshooting in distribution \\ systems, using the service quality meters electricity
}

Artículo recibido: enero de 2011

Artículo aceptado: julio de 2011

Víctor A. Gómez

Robin A. Peña $a^{* * *}$

César Hernández $z^{* * *}$

\begin{abstract}
Resumen
Este artículo presenta una nueva metodología para la identificación y localización de fallas en sistemas de distribución de energía eléctrica, por medio de la implementación de medidores electrónicos que registren la continuidad en el servicio eléctrico. La implementación de los registradores, facilita detectar fallas permanentes o transitorias en redes no homogéneas, proporcionando información sobre puntos débiles de los sistemas de potencia, para luego poder adoptar medidas correctivas con respecto a la duración y frecuencia de las interrupciones, en los circuitos de los sistemas de distribución local.

A través de este mecanismo de regulación de los sistemas desde la generación, comercialización, distribución y operación de la electricidad, se plantea una solución que permita mitigar la discontinuidad del servicio y los cuantiosos costos que producen a los agentes del sector eléctrico y, principalmente, a los usuarios de la energía eléctrica.
\end{abstract}

\section{Palabras clave}

Interrupción eléctrica, continuidad eléctrica, sistemas de distribución, calidad de la energía eléctrica, medidor

* Este artículo presenta resultados del proyecto investigativo del grupo Armos, titulado: "Diseño y construcción de un prototipo de medición de los indicadores de la calidad del servicio de energía eléctrica (DES y FES) para usuario residencial".

** Tecnólogo en Electricidad de la Universidad Distrital Francisco José de Caldas y estudiante de Ingeniería en Eléctrica por ciclos propedéuticos de la misma universidad. Corre: victorgomez8807@gmail.com

*** Tecnólogo en Electricidad de la Universidad Distrital Francisco José de Caldas y estudiante de Ingeniería en Eléctrica por ciclos propedéuticos de la misma universidad. Correo: alejandro2905@gmail.com

****Ingeniero Electrónico y docente de la Universidad Distrital Francisco José de Caldas. Magíster en Ciencias de la Información y las Comunicaciones de la misma universidad. Correo: cahernandezs@udistrital.edu.co 


\section{Abstract}

This paper presents a new methodology for identifying and locating faults in distribution systems of electric power, through the implementation of electronic meters that record the continuity of electric service. The implementation of the meters, to provide detection permanent or transient fault in non-homogeneous networks and providing information of weaknesses in the power system and then to take corrective action with respect to the duration and frequency of breaks in the circuits local distribution systems.

By means of this mechanism regulation of the systems from the generation, marketing, distribution and operation of electricity. This raises a solution to mitigate the discontinuity of service and significant costs to produce electricity sector agents and mainly to electricity users.

\section{Keywords}

Power outage, electrical continuity, distribution systems, electrical power quality, meter electronic.

\section{Introducción}

A partir de la expansión de los sistemas de distribución de energía eléctrica en el mundo, el problema de la discontinuidad en el servicio se ha convertido en un tema de gran interés para los agentes del sector eléctrico (en las áreas de planeación, operación, distribución) y especialmente para los usuarios residenciales e industriales. Tanto las empresas prestadoras del servicio de energía eléctrica como los usuarios, interactúan con respecto a la valoración de los costos debidos a las interrupciones del suministro de energía eléctrica (Peñuela, A., Pacheco, J., Jairo, H., Ordóñez, G., IEEE. \& Benjamín. J., 2005).

Con los procesos de globalización, las empresas prestadoras del servicio de electricidad del mundo realizan estudios para valorar los costos debidos a una inadecuada continuidad en el suministro. De esta forma, pueden obtener un punto equidistante entre el costo de inversión para mejorar la confiabilidad de un sistema y los costos que las inte- rrupciones representan a los usuarios del servicio (Wacker \& Billinton, 1989).

Vale la pena recordar que la localización de fallas no es un problema nuevo. Sin embargo, las investigaciones que se han realizado en esta área recaen sobre los sistemas de transmisión, donde las características homogéneas de la línea, la medición en ambos terminales y la disponibilidad de más equipos, permiten localizar el sitio de falla con alta precisión. La localización en sistemas de distribución es más problemática, por la complejidad que produce la presencia de conductores no homogéneos, cargas intermedias, laterales y desbalance del sistema y de la carga (Morales, Barrera, Vargas, 1989: 919-930). Lo anterior, evidencia que un sistema de distribución local es más vulnerable a los diferentes factores que afecten la continuidad del servicio prestado, por la singularidad de las topologías de sus redes eléctricas.

A partir de la investigación desarrollada, se busca presentar el método de implementación de medidores electrónicos, los cualespermiten tener un mejor esquema de regulación y registro de los sistemas de potencia, para mitigar la incidencia de factores como la discontinuidad en el servicio eléctrico.

\section{Historia}

Los estudios sobre continuidad o confiabilidad en los sistemas eléctricos han estado enfocados en las áreas de generación y cogeneración, donde se destaca la homogeneidad de los sistemas de potencia. Sin embargo, desde 1960, han existido algunos pocos estudios para la determinación de la confiabilidad tanto en transmisión y distribución como en los puntos de carga (Baeza, Rodríguez\& Hernández, 2003: 33-39). Tales estudios, han representado el diseño de metodologías a partir de algoritmos de identificación de fallas, basados en el historial de eventos ocurridos y en lo establecido por los entes de regulación.

Desde mediados del siglo XX hasta nuestros días, el sector eléctrico del mundo ha crecido de ma- 
nera exponencial. Por ejemplo, las empresas distribuidoras de Estados Unidos experimentaron el comienzo de un rápido aumento en el número de usuarios de energía eléctrica. Este crecimiento de la demanda, provocó la expansión de los sistemas de planeación y distribución. A medida que los sistemas de distribución crecieron en complejidad, la continuidad del servicio se volvió un tema de gran importancia para los ingenieros eléctricos, y, más aún, cuando el 14 de agosto del 2003, Estados Unidos y Canadá experimentaron el mayor apagón de la historia (con una duración aproximada de 29 horas) que afectó a cerca de 50 millones de personas. Se estima que las pérdidas fueron más de \$USD 5.000 millones.

Se calcula que el costo de los apagones que actualmente sufre Estados Unidos llega a los \$USD 120.000 millones. En virtud de lo cual, el plan energético que planteó el presidente George W. Bush, buscaba ahorrar energía mediante el uso adecuado de la tecnología, además de presentar recomendaciones para modernizar el sistema eléctrico de dicho país (Torres, Acero, Flechas, Saucedo \& Quintana, 2003).

Con todas las experiencias vividas en el mundo con respecto a las deficiencias en la calidad de la energía eléctrica, organizaciones internacionales como la International Electrotechnical Commission (IEC) y el Institute of Electrical and Electronics Engineers (IEEE) desarrollaron una serie de normas y métodos de valoración directa de costos debidos a interrupciones ${ }^{1}$.

\section{Confiabilidad en los sistemas de distribución}

Mejorar la calidad del servicio prestado es de vital importancia para la sociedad actual, en donde la energía eléctrica es indispensable en la mayoría de las actividades del ser humano, tanto en labores cotidianas domésticas como industriales. Con el avance de las tecnologías, la mayoría de equipos eléctricos y electrónicos de uso doméstico e indus-

1 Ver estudios de Dialynas, Megaloconomus \& Daly (2001); Billinton, Subramaniam \& Wacker (1993); Sullivan, Vardell, Suddeth \& Vojdani (1995) y Sousa Martins, Martins, Member, Alegria \& Pires (2003). trial son más vulnerables a variaciones de los parámetros de alimentación. Por ende, la calidad de la energía y la continuidad en el servicio de suministro, deben regirse bajo principios de confiabilidad.

En consecuencia es necesario adoptar metodologías que faciliten la rápida y confiable identificación de fallas y, de esta manera, establecer medidas que mitiguen el impacto económico y social, asociado a los factores característicos de la energía eléctrica.

Las inversiones que hacen los operadores de red y los comercializadores para mejorar dicha calidad del servicio, se debe evidenciar de manera clara y transparente en interrupciones menos frecuentes y prolongadas, plasmadas en datos de indicadores veraces y definitivos.

\section{Actual identificación y localización de fallas en sistemas de distribución}

El diagnóstico de fallas en las redes de distribución es de gran importancia, incluso en las redes de media tensión. Del mismo modo, la identificación y localización de tales eventos es un importante factor para la calidad del servicio.

Para el diagnóstico de fallas se emplean algoritmos que consisten principalmente en el cómputo de la impedancia de la línea de falla, basados en el voltaje y la corriente registrados directamente en el alimentador primario. Por lo tanto, se establece una relación entre la impedancia y la distancia de la falla, teniendo presente los parámetros de la línea (Sullivan, Vardell \& Johnson, 1996: 23-25). Este método es eficiente para los sistemas de transmisión porque permite detectar, en un punto preciso, la ocurrencia de la interrupción; sin embargo, tal algoritmo no es aplicable a redes de distribución, debido a que éstas presentan topologías mucho más complejas, así, por ejemplo, la distancia asociada con la impedancia estimada puede coincidir con diferentes puntos de un sistema ramificado.

\section{Índices establecidos en Colombia}

Actualmente las empresas suministradoras del servicio de energía tienen un valor máximo admisible 


\begin{tabular}{|c|c|c|c|c|c|c|c|c|c|c|}
\hline \multirow[t]{2}{*}{ Grupo' } & \multicolumn{2}{|c|}{ Índices Anuales } & \multicolumn{2}{|c|}{$\begin{array}{ll}\text { Enero, } & \text { Febrero, } \\
\text { Marzo } & \\
\end{array}$} & \multicolumn{2}{|c|}{ Abril, mayo, junio } & \multicolumn{2}{|c|}{$\begin{array}{l}\text { Julio, agosto, sep- } \\
\text { tiembre }\end{array}$} & \multicolumn{2}{|c|}{$\begin{array}{l}\text { Octubre, noviem- } \\
\text { bre, diciembre }\end{array}$} \\
\hline & DES & FES & DES & FES & DES & FES & DES & FES & DES & FES \\
\hline 1 & 11 & 23 & 2.7 & 7 & 3.2 & 7 & 2.9 & 6 & 2.2 & 6 \\
\hline 2 & 19 & 44 & 5.0 & 12 & 6.0 & 12 & 4.5 & 10 & 3.5 & 10 \\
\hline 3 & 29 & 51 & 7 & 10 & 9 & 16 & 7 & 15 & 6 & 10 \\
\hline 4 & 39 & 58 & 9 & 13 & 12 & 17 & 11 & 16 & 7 & 12 \\
\hline
\end{tabular}

Tabla 1. Indicadores de calidad 2010 (valores máximos admisibles) [11]

Fuente: REPORT. Medellin utilities E.S.P. In compliance with Act 142 of 1994 and the CREG resolutions 058 and 119 of 2000 and 078 of 2007.2010 maximum allowable values.

de interrupciones, que pueden utilizar a lo largo del año para trabajos de mantenimiento y expansión de las redes de distribución. Para el caso de Latinoamérica, Colombia estableció para el año 2010 los índices máximos admisibles en la duración y frecuencia de las interrupciones. En la tabla 1 se describen los índices para cada trimestre del año.

Si la empresa prestadora del servicio supera los valores máximos admisibles, deberá indemnizar a los usuarios por la falta en la prestación del servicio público.

\section{Esquema de monitorización cabecera del circuito}

En la fig. 1 se logra observar un diagrama del sistema de distribución, en el cual la subestación principal o alimentador primario (1) está encargada de suplir energía eléctrica a usuarios residenciales $(2$ y 3 ) e industriales (8), a través de líneas de distribución $(4,5$ y 6$)$ y de un nodo de interconexión (7). Los operadores de red miden la continuidad del servicio de energía directamente sobre la subestación (1). Entonces, si ocurre una falla en los puntos (4, 5, 6 ó 7), esta interrupción no será oportunamente registrada por los medidores de la subestación, debido a que estos solo monitorizan los interruptores que se encuentran alojados en la misma. En consecuencia, los usuarios residenciales e industriales serán los principales afectados por la discontinuidad del servicio, que repercutirá en altas perdidas en productividad, daño en equipos, productos y procesos. Todo lo anterior, es causadopor la tardanza en la identificación y localización de la falla eléctrica para su posterior re conexión.

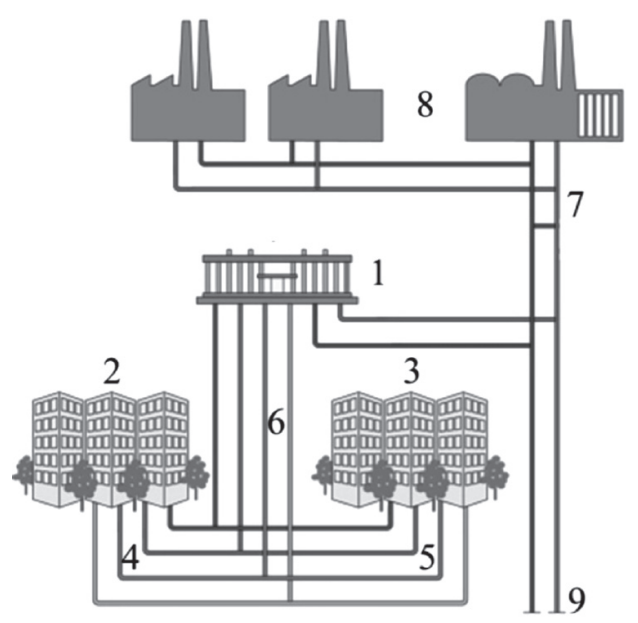

Figura 1. Diagrama del sistema de distribución

1: Subestación principal en la red de distribución

2 y 3: Usuarios residenciales

4, 5 y 6: Red eléctrica de distribución

7: Nodo de conexión usuarios industriales

8: Usuarios industriales

9: Red principal de distribución 
Se estima que anualmente Estados Unidos tiene pérdidas superiores a los 25 billones de dólares, debido a las interrupciones en el servicio de energía, causadas por fenómenos naturales o por una mala coordinación y supervisión de los sistemas de transmisión y distribución (Torres, et al., 2003).

\section{Metodología propuesta para la localización de fallas en sistemas de distribución}

Frente a la problemática planteada en relación a la identificación y localización de fallas en sistemas de distribución, el grupo de investigación de "Arquitecturas modernas para sistemas de alimentación-ARMOS”, en conjunto con la Universidad Distrital Francisco José de Caldas, desarrollaron un primer prototipo electrónico con el nombre de Medidor de indicadores de calidad en el servicio de energía eléctrica (DES y FES) para usuario residencial (Gómez, Peña \& Hernández, 2010: 101-112), concebido inicialmente como un registrador enfocado a la regulación de los sistemas desde los usuarios suscriptores del servicio energético.

La funcionalidad del medidor se ha ampliado de forma progresiva, mediante la incorporación de nuevos esquemas de medición, algoritmos de control y sistemas de comunicación. Para orientar, de manera más general, a la solución de problemas de electrotecnia, enfocados hacia la calidad de la potencia eléctrica y del servicio.

\section{Instalación de medidores electrónicos}

La gran mayoría de los sistemas de distribución poseen topologías de tipo radial. Por tanto, es necesario disponer de medidores electrónicos en diferentes puntos del sistema de conexión. Los nodos de análisis pueden ser: transformadores de media y baja tensión, interruptores de potencia, barrajes, entre otros. Entre más puntos se tomen del sistema eléctrico para la identificación y localización de fallas, mayor será la precisión en el diagnóstico de discontinuidad del servicio energético.

Igualmente, los medidores electrónicos pueden ser implementados directamente en usuarios residenciales e industriales. De ser este el caso, los medidores deben ser instalados directamente en el armario que contiene los registradores de energía activa, específicamente en el barraje de alimentación de los diferentes circuitos. En la fig. 2 y 3 se observa la instalación.

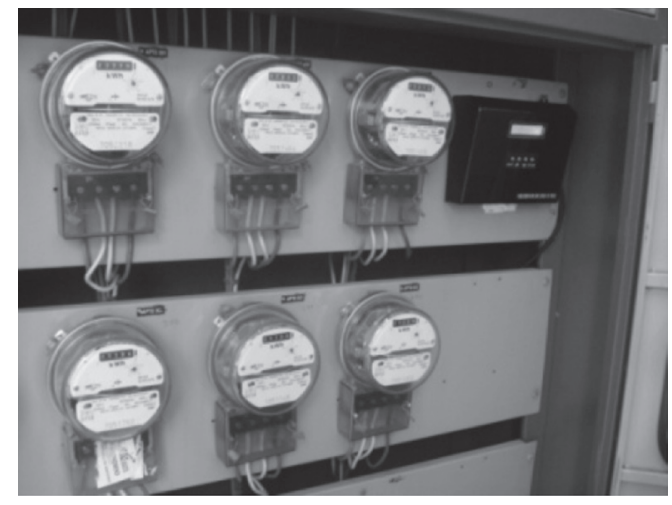

Figura 2. Armario de medidores

Fuente: elaboración propia

El acondicionamiento del sensor de voltaje que se encuentra alojado en el medidor electrónico, depende del nivel de tensión del nodo de interconexión de la red de distribución local donde será instalado el prototipo; por lo que se pueden encontrar diferentes niveles de tensión.

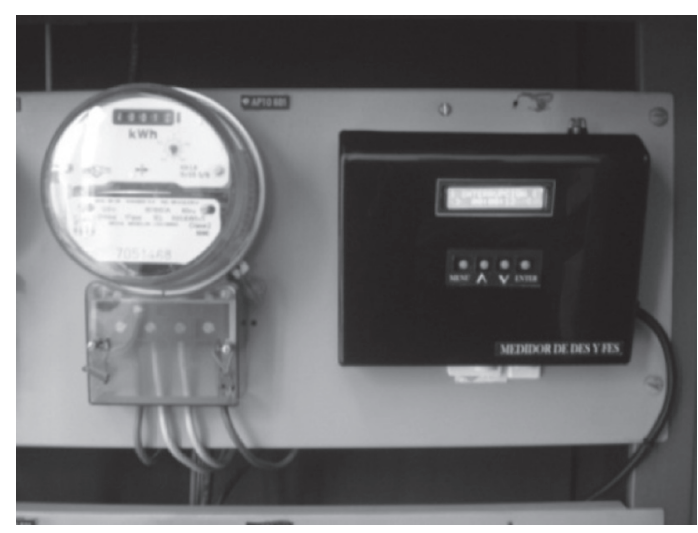

Figura 3. Medidor de energía activa y medidor de continuidad en el servicio de energía eléctrica

Fuente: elaboración propia

La instalación de los medidores debe realizarse por personal calificado, cumpliendo las normas nacio- 
nales e internacionales. Para el caso colombiano, la instalación se debe regir bajos los principios establecidos en la Norma Técnica Colombiana 2050 (NTC 2050) y el Reglamento técnico de Instalaciones Eléctricas (RETIE).

\section{Detección de eventos de tensión}

Se debe censar la componente fundamental de la señal de voltaje al momento de prefalla y falla, debido a que pueden existir interrupciones permanentes o transitorias.

En este orden de ideas, el colapso de una falla presente en el sistema de distribución local, ocasiona cuantiosas pérdidas económicas a los pequeños y grandes consumidores. Por tal razón, el medidor electrónico censa la usencia de tensión e informa a los especialistas para que se genere un rápido aislamiento, reparación y restauración del servicio energético. Con lo anterior, se mitigarían los altos índices de la duración de las interrupciones presentes en un circuito (DES).

Por otro lado, las interrupciones transitorias no se pueden identificar por simple inspección. Por tal razón, el medidor electrónico permite detectar los puntos débiles de los sistemas de potencia en donde reincide la falla. De esta manera, se logra tener un esquema de regulación para atenuar los altos índices del indicador de frecuencia equivalente en las interrupciones de un circuito (FES).

\section{Sistema de comunicación}

A partir de la necesidad de obtener los registros de los medidores en los diferentes puntos de la red de distribución, se debe implementar un sistema de transmisión inalámbrico, adecuado para dar soporte a las necesidades de telemetría. Tal comunicación es establecida mediante estándares ZigBee, por su bajo coste, soporte para múltiples topologías de red, rango de alcance y bajo consumo de energía.

Este sistema de comunicación permite obtener un estudio en tiempo real de la información adquirida por el medidor electrónico, frente a un suceso ca- racterístico en las líneas de distribución. Por tanto, el medidor emite un diagnóstico que informa a los especialistas (principalmente a los operadores de las subestaciones) de los eventos de ocurrencia en las redes de distribución local.

Una vez emitido un diagnóstico por el medidor, el receptor de la información -ubicado en la subestación principal- migrará los datos recibidos hacia el ordenador de la subestación, a través del software que se observa en la fig. 4. Seguidamente, este programa generará un reporte de la ocurrencia de los eventos con fecha y hora, para luego emitir la identificación de la línea que ha salido de servicio. Con base en este informe, el operador o el especialista de turno, podrá emitir un protocolo a seguir para proceder con la recuperación del sistema de distribución local. Es decir, restablecer el servicio de energía para los usuarios.

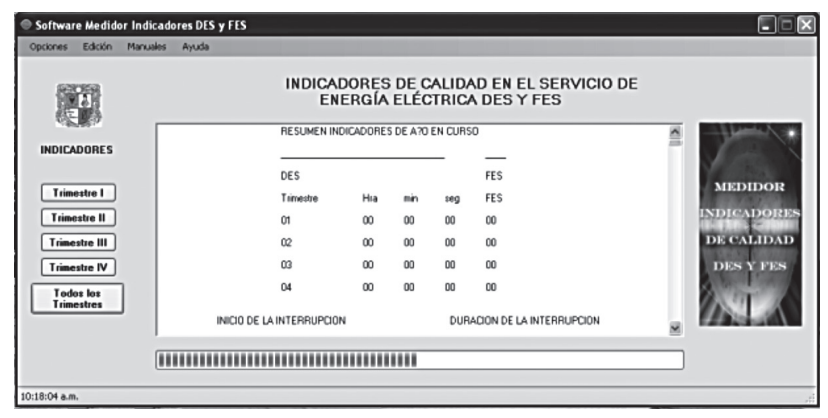

Figura 4. Software «DES y FES»

Fuente: elaboración propia

Dentro de la regulación colombiana, está establecido que los operadores de red y los comercializadores de la energía eléctrica deben generar reportes de los indicadores DES y FES de manera trimestral, para ser presentados a organismos de control y regulación. En relación a lo anterior, el software cuenta con la posibilidad de observar el historial de ocurrencia de eventos con información referente a la duración, fecha y localización de la falla en el sistema de los últimos 12 meses. Por tanto, el programa permite tener una mejor inspección de las características de las interrupciones en los circuitos. 


\section{Conclusiones}

El nuevo esquema de regulación de la energía eléctrica y la competencia de los mercados nacionales e internacionales, ha hecho que las empresas prestadoras del servicio de energía eléctrica adopten nuevas metodologías para el mejoramiento del servicio prestado hacia usuarios de sistemas de distribución industrial, comercial, urbano y rural.

La implementación de medidores que registren la continuidad del servicio de energía, permite tener un mejor control e inspección de los sistemas de distribución. Así mismo, facilita la oportuna y rápida localización de fallas, mitigando los elevados costos de compensaciones, hechas por los diferentes agentes del sector eléctrico, a causa de la inadecuada continuidad en el suministro de energía. Todo lo anterior, a fin de remediar la ausencia del servicio energético en los usuarios finales.

La adopción de nuevas metodologías para la identificación de fallas en sistemas de distribución, partiendo de sistemas expertos de monitorización, permite aminorar los indicadores de duración y frecuencia de las interrupciones del servicio eléctrico.

Igualmente, el correcto diagnóstico de fallas en las redes eléctricas, permiteevitar colapsos en los sistemas de transmisión y distribución de electricidad. Además, fomenta a los usuarios y agentes del sector eléctrico para que sean protagonistas en el mejoramiento del sector energético del mundo.

\section{Agradecimientos}

Agradecemos al Centro de Investigaciones y Desarrollo Científico (CIDC) de la Universidad Distrital Francisco José de Caldas, quien financió este proyecto y brindo todas las herramientas necesarias para la realización del mismo.

\section{Bibliografía}

Baeza, R., Rodríguez, J.\& Hernández. J. (2003). "Evaluación de confiabilidad de sistema de distribución eléctrica en desregulación". En
Revista Facultad de Ingeniería, vol. 1, núm. 1.Chile: Universidad de Tarapacá.

Billinton, R.,Subramaniam, R. \& Garry, W. (1993). “Understanding Industrial Losses Resulting from Electric Service Interruptions". En IEEE Transactions on Industry Applications, vol. 29, núm. 1.Canadá, Saskatoon: Saskatchewan.

Dialynas, E., Megaloconomus, S.\& Dali, V. (2001)."Interruption Cost Analysis for the Electrical Power Customers in Greece".En $\mathrm{Na}$ tional Technical University of Athens. Athens, Greece.

Gómez, V., Peña, R.\&Hernández, C. (2010).“Diseño y construcción de un medidor de indicadores de calidad en el servicio de energía eléctrica (DES y FES) para usuario residencial". En Revista Lasallista de Investigación, vol. 7,núm. 2. Universidad de la Salle.

Morales, G., Barrera, R. \& Vargas H. (2009).“Ubicación única de fallas en sistemas de distribución por medio de zonas con SVM" [en línea]. EnRevista Facultad de Ingeniería, núm. 47. Colombia: Universidad de Antioquia, disponible en http://redalyc.uaemex.mx/src/inicio/ ArtPdfRed.jsp?iCve $=43004717$

Peñuela, A., Pacheco, J., Jairo, H., Ordóñez, G., IEEE. \&Benjamín. J. (2005).“Methodological proposal for cost valuation due to an inadequate continuity in the electric energy supply".Bogotá, III International Symposium on Electric Energy Quality SICEL, 2005.

REPORT: Medellin utilities E.S.P. In compliance with Act 142 of 1994 and the CREG resolutions 058 and 119 of 2000 and 078 of 2007. 2010 maximum allowable values.

Sousa, L., Martins, F.,Member IEEE.,Alegria, C.,\&Pires, V. (2003)."A network distribution power system fault location based on neuronal eigenvalue algorithm".

Sullivan, M.,Vardell, T. \& Johnson, M. (1996)."Power interruption costs to industrial 
and commercial consumers of electricity". Charlotte, USA.

Sullivan, M.,Vardell, T.,Suddeth, N. \&Vojdani, A. (1995)."Interruption costs, customer satisfaction and expectations for service reliability". En IEEE Transactions on Power Systems, vol. 11, núm. 2. Charlotte, USA.

Torres, H., Acero, G., Flechas, J., Saucedo, J.\& Quintana, C. (2003). "Electric power, a quality product”. Bogotá: ICONTEC, 2003. ISBN 958-9383-38-6.
Barrera, V., Cormane, J., Rodríguez, J., Santos, G., Carrillo, G., Ordóñez, G., Vargas, H.\& Mora, J. (2005). "Methodology for improving the indexes of continuity in the electric energy supply".Bogotá, III International Symposium on Electric Energy Quality SICEL, 2005.

Wacker, G.\&Billinton, R. (1989).“Customer cost of electric service interruptions".En Proceedings of the IEEE,vol. 77, núm.6. Canadá. 